\title{
VOIR EN ART, VOIR EN SCIENCES
}

Maria Giulia Dondero

Presses Universitaires de France | « Nouvelle revue d’esthétique »

2016/1 n 17 | pages 139 à 159

ISSN 1969-2269

ISBN 9782130792505

Article disponible en ligne à l'adresse :

http://www.cairn.info/revue-nouvelle-revue-d-esthetique-2016-1-page-139.htm

\section{Pour citer cet article :}

Maria Giulia Dondero, «Voir en art, voir en sciences », Nouvelle revue d'esthétique 2016/1 (n¹7), p. 139-159.

DOI 10.3917/nre.017.0139

Distribution électronique Cairn.info pour Presses Universitaires de France.

(c) Presses Universitaires de France. Tous droits réservés pour tous pays.

La reproduction ou représentation de cet article, notamment par photocopie, n'est autorisée que dans les limites des conditions générales d'utilisation du site ou, le cas échéant, des conditions générales de la licence souscrite par votre établissement. Toute autre reproduction ou représentation, en tout ou partie, sous quelque forme et de quelque manière que ce soit, est interdite sauf accord préalable et écrit de l'éditeur, en dehors des cas prévus par la législation en vigueur en France. Il est précisé que son stockage dans une base de données est également interdit. 


\section{MARIA GIULLA DONDERO}

\section{Voir en art, voir en sciences [l]}

Le présent article porte sur les dispositifs visuels qu'on appelle généralement «métavisuels » ou « métasémiotiques » et qui ont été formulés pour analyser notamment l'image artistique. Nous allons ici explorer la possibilité d'utiliser ces dispositifs pour analyser les images scientifiques et proposer des ajustements éventuellement requis. Est-il possible d'envisager une telle transposition ? Ces dispositifs sont-ils utiles pour comprendre la rhétorique de la visualisation dans les sciences contemporaines?

Pour tester cette transposition entre les images artistiques et les images scientifiques, je m'appuierai en premier lieu sur les travaux d'un théoricien de l'image artistique proche des théories sémiotiques, Victor Stoichita, et en deuxième lieu, sur les travaux du philosophe et sémioticien Jean-François Bordron. Je tâcherai de faire rencontrer ces travaux tout au long de mon texte : le travail d'un théoricien de l'image qui a forgé des instruments pour comprendre le fonctionnement des images à travers des dispositifs métavisuels (notamment le cadre dans le cadre), et celui de Jean-François Bordron consacré à la notion de méréologie, à savoir les rapports entre totalité et parties ${ }^{[2]}$. La question de la composition/décomposition de la totalité est évidemment centrale dans la réflexion de Stoichita sur le cadre dans le cadre, bien qu' elle ne soit pas exposée dans ces termes.

Parmi les nombreux ouvrages de Stoichita, L'Instauration du tableau ${ }^{[3]}$ et l'Eil mystique ${ }^{[4]}$ abordent les rapports que chaque image en tant que totalité articulée entretient avec ses parties et sur les relations que chaque partie noue avec les autres parties au sein de cette totalité. Cette question est à la base de l'ouvrage L'Instauration du tableau, consacré aux rapports entre la topologie de la peinture religieuse et les genres dérivant de cette dernière à l'époque moderne. Dès les premières pages de l'ouvrage, Stoichita affirme que la peinture religieuse est à la source des différents genres qui se développent au quinzième siècle (portrait, nature morte, paysage, etc.) ; plus particulièrement, il montre la manière dont, à l'époque moderne, ces
1. Je tiens à remercier chaleureusement Marion Colas-Blaise pour la précieuse relecture de ce texte.

2. Jean-François Bordron, " L'objet en parties (esquisse d'ontologie matérielle) ", Langages, 1991, n 103 , pp. 51-65.

3. Victor Stoichita, L'Instauration du tableau. Métapeinture à l'aube de temps modernes, Paris, Méridiens-Klincksieck, 1993.

4. Victor Stoichita, L'Eil mystique. Peindre l'extase dans l'Espagne du siècle d'or, Paris, Le Félin, 2011. 
genres prennent leur autonomie par rapport au tableau religieux. Cette autonomisation est la résultante d'opérations de déplacement de frontières, ainsi que de stratégies d'encadrement qui transforment les marges du tableau religieux en des centres de nouvelles images de genre. Ce sont le cadre, l'encadrement, le croisement de cadrages et les superpositions d'encadrement qui caractérisent les différents dispositifs métapicturaux (fenêtre, niche, tableau, miroir, porte, carte géographique, parois) qui sont au cœur des réflexions de Stoichita sur les marges, le montage, l'intertexte visuel. Ce qu'il est important de rappeler est que chacun de ces dispositifs métapicturaux est lié à des opérations qui consistent à focaliser l'attention, additionner les points de vue, soustraire la vision, etc., autant de procédés que nous allons examiner dans le détail.

\section{LES DISPOSITIFS MÉTAPICTURAUX SELON VICTOR STOICHITA}

Le dispositif pivot exploré par Stoichita, le cadre, concerne les opérations qui permettent de concentrer l'attention, de construire un centre et une périphérie de l'image, de distinguer des forces englobantes et des forces englobées, ainsi que de distribuer les points d'appui sur lesquels se fondent la structure des images et notre cheminement perceptif. Les opérations fondamentales engendrées à travers le dispositif du cadre sont principalement au nombre de trois : focaliser/centrer/distribuer les centres d'attention.

Passons en revue les dispositifs décelés par Stoichita afin de les associer à quelques opérations fondamentales.

1. Le tableau dans le tableau. Il concerne les opérations du montage, c'est-à-dire de rapprochement, d'inclusion, de liaison et de déliaison. Ces opérations rhétoriques de rapprochement, d'éloignement, d'inclusion, de mise entre guillemets, visibles par exemple dans les cabinets de curiosités étudiés par Stoichita ${ }^{[5]}$, mettent en scène la manière dont un tableau peut réfléchir sur l'histoire de l'art, voire la commenter. Dans le cas de la peinture religieuse, et notamment dans les tableaux des visions, étudiés par Stoichita dans l'Eil mystique, on peut repérer d'autres fonctions du cadre dans le cadre : notamment la duplication et la hiérarchisation des niveaux de réalité

5. Un des exemples donnés par Stoichita est le tableau de David Teniers le Jeune, Le Gouverneur Léopold-Guillaume et sa collection de tableaux à Bruxelles, Vienne, vers 1650 .

6. Un des exemples donnés par Stoichita est le tableau d'Alonso Cano, Vision de Saint Bernard, Madrid, 1650. tels que le niveau immanent de la représentation (le terrestre) et le niveau transcendant de la représentation (le céleste) ${ }^{[6]}$.

Retenons enfin que le tableau dans le tableau spécifie les opérations de focalisation et de centration valorisées par le cadre et particulièrement l'action de croiser les perspectives, d'encastrer les points de vue l'un dans l'autre et, dans certains cas, de les hiérarchiser. 
2. Le dispositif de la fenêtre. Il permet de valoriser des actions telles que " dépasser ", " aller au-delà », " projeter au loin ", " conquérir la distance », « apprivoiser le lointain » qui ont accompagné la naissance du genre paysage. Les opérations que nous retenons comme spécifiques du cadre-fenêtre sont donc celles de la projection, de l'exploration, du devancement et de l'anticipation.

3. La niche. Ce dispositif métavisuel valorise des opérations caractérisées par des forces contraires telles que /bloquer la vision vers l'horizon/ et / envahir l'espace du spectateur/ jusqu'à ce que ce dernier retourne l'attention sur lui-même, presque comme devant un miroir. La niche est d'ailleurs le dispositif utilisé dans les natures mortes et dans les Vanitas qui, tout en apparaissant comme des creusements dans la banalité des objets quotidiens, fonctionnent comme des appels à la conscience du spectateur à travers deux moyens : d'un côté, la noirceur de la profondeur et le mur, qui bloquent toute ouverture vers l'horizon; de l'autre, les objets en train de tomber en deçà du seuil de séparation entre l'image et l' espace d'observation, de l'autre ${ }^{[7]}$. Nous pouvons ainsi retenir comme opérations caractérisant le fonctionnement de la niche l'obstruction de l'horizon du regard de l'observateur contrebalancée par l'envahissement de l'espace de l'observateur.

4. Le miroir. Ainsi que toutes les surfaces réfléchissantes présentes dans l'image (armures, verres, bouteilles, couverts en or et en argent, etc.), il permet le retour du regard sur la production du tableau et se manifeste comme une sorte de réflexion sur les activités de produire et de regarder ${ }^{[8]}$. Mais, à la différence de la niche, le miroir ne bloque pas le regard vers l'horizon : il permet au contraire de renverser la profondeur de l'horizon en avant-plan de l'image - et d'inclure la figure du peintre dans le tableau. Le miroir fait en sorte que le producteur se réfléchisse à l'intérieur de son produit. Nous pourrions ainsi concevoir le miroir en tant que dispositif d'encadrement permettant de renverser la vision ainsi que d'additionner les points de vue à l'intérieur de l'image en englobant les scènes qui lui seraient a priori extérieures.

5. La porte ainsi que les rideaux. Ils favorisent quant à eux l'observation de biais, le regard qui dépasse l'obstacle qui s'infiltre, voire le regard entredeux ${ }^{[9]}$. Portes et rideaux permettent de révéler et de cacher, mais surtout d'entrevoir, sans se faire remarquer : c'est la faille dans la fermeture du champ de vision qui est en jeu ici.

Par rapport à l'ouverture assurée par la fenêtre, qui est toujours une ouverture vers l'autre et le lointain, la porte entrouverte ou le rideau semitiré sont des ouvertures vers le proche et vers l'intérieur. Chaque image représentant des portes ou des rideaux met en scène différentes manières
7. Un des exemples donnés par Stoichita est le tableau de Juan Sánchez Cotán, Coing, chou, melon et concombre, San Diego, 1602, où le miroir sphérique permet de voir le peintre à son chevalet avec une femme (un modèle?) à ses côtés.

8. Un des exemples donnés par Stoichita est le tableau de Simon Luttichuys, Vanité avec crâne, 1610-1661.

9. Un des exemples donnés par Stoichita est le tableau de Nicolas Maes, L'écouteuse, Londres, 1655. 
d'ouvrir ou de fermer le champ de vision offert par le cadrage global du tableau. Les opérations pertinentes peuvent dès lors précisément se formuler comme suit : soustraire et/ou parcelliser la vision, d'un côté, dénicher les stratégies d'insertion du regard dépassant les obstacles, de l'autre.

Ces opérations peuvent se résumer comme suit :

1. CADRE : focaliser/centrer/distribuer les centres de l'attention;

2. IMAGE DANS L'IMAGE : croiser les visions/encastrer les points de vue;

3. FENÊTRE : projeter/explorer/devancer;

4. NICHE : bloquer le regard vers l'horizon/envahir l'espace de l'observateur;

5. MIROIR : renverser la vision/additionner les points de vue;

6. PORTE/RIDEAUX : soustraire/parcelliser la vision/dénicher des stratégies d'insertion du regard.

\section{TRANSPOSITION D'OPÉRATIONS VERS LES IMAGESSSIENTIFIQUES}

Après ce bref aperçu des opérations rhétoriques mises en valeur par les dispositifs métapicturaux, demandons-nous si ces opérations peuvent être utiles pour comprendre les images scientifiques contemporaines. Est-il pertinent de mettre ces opérations méréologiques à l'épreuve des images scientifiques de l'époque moderne, contemporaines des tableaux sur lesquels Stoichita appuie sa théorie de la métapeinture ? Le choix d'un corpus d'images scientifiques contemporaines se fonde sur le fait que ces dispositifs fonctionnent comme les catégories de " classique » et de « baroque » chez Heinrich Wölfflin ${ }^{[10]}$ - ensuite réutilisées en sémiotique par Claude Zilberberg ${ }^{[11]}$ et Jean-Marie Floch ${ }^{[12]}$ pour analyser des objets distants de la peinture tels que des styles vestimentaires. Ces catégories sont en effet transhistoriques et régissent les compositions visuelles de tout temps et de tout statut. Certes, dans le cadre de notre recherche, il faut les soustraire de leur lien avec la spécificité artistique et trouver un niveau de comparaison suffisamment abstrait pour que la transposition des arts aux sciences puisse fonctionner.

10. Heinrich Wölfflin, Principes fondamentaux de l'histoire de l'art. Le problème de l'évolution du style dans l'art moderne, Monfort, 2002 (Gallimard, 1966).

11. Claude Zilberberg, La Structure tensive, Liège, Presses universitaires de Liège, 2012.

12. Jean-Marie Floch, Les Formes de l'empreinte: Brandt, Cartier-Bresson, Doisneau, Stieglitz, Strand, Périgueux, P. Fanlac, 1987 ; Identités visuelles, Paris, Puf, 1995 (réed. 2010).

13. Lorraine Daston et Peter Galison, Objectivité [2007], Paris, Les presses du réel, 2012.

nouvelle Revue d'esthétique n $17 / 2016$
Du côté de l'histoire des sciences, nous avons appris de l'ouvrage Objectivité de Lorraine Daston et Peter Galison ${ }^{[13]}$ qu’on peut repérer trois étapes et trois épistémès de la représentation des phénomènes scientifiques qui se succèdent : "La vérité d'après nature " au XVIII ${ }^{e}$ siècle, "L'objectivité mécanique " au $\mathrm{XIX}^{\mathrm{e}}$ siècle, le "Le jugement exercé " au $\mathrm{xx}^{\mathrm{e}}$ siècle. Cet ouvrage permet de comprendre que le problème de l'objectivité a toujours été une question de cadrage : pendant la première époque, il s'est agi de rendre compte d'un type isolé d'occurrences ; pendant la deuxième époque, de rendre compte de la diversité et de l'exception; dans la troisième époque, de rendre compte de la multiplicité et de son organisation par patterns et par ressemblances de famille. Il s'agit dans tous les cas d'un problème de méréologie, voire de construction de la totalité d'un 
Voir en art, voir en sciences

objet (par compression, par soustraction, par superposition, par addition, par exclusion, etc.) et de ses composantes.

Nous allons à présent voir si les opérations sélectionnées par Stoichita peuvent être repérées dans les images scientifiques et la manière dont elles fonctionnent dans différents domaines de la recherche contemporaine (la biologie moléculaire, l'astrophysique, l'archéologie).

\section{L'image scientifique et la « totalité d'objet »}

Une première distinction générale est nécessaire avant de commencer notre exploration sur les images scientifiques. Si chaque tableau examiné par Stoichita est une totalité autographique ${ }^{[14]}$, unique et originale - son statut d'objet d'art en fige et sacralise chaque trait et le rend non modifiable -, l'objet scientifique visualisé, en revanche, est toujours produit à travers différentes chaines d'images qui se déterminent et se spécifient mutuellement et, surtout, sont continuellement remises en cause, voire falsifiées.

On pourrait partir de l'hypothèse qu'aucune image scientifique ne correspond à un objet scientifique dans sa totalité mais que plusieurs images peuvent, ensemble, construire une approximation ${ }^{[15]}$ afin de rendre compte d'un fonctionnement que nous pourrions appeler une totalité - qui, en sciences expérimentales, est toujours provisoire. Ce n'est en fait que dans les ouvrages et dans les articles de vulgarisation que nous sommes face à la présentation d'une image ayant la prétention de mettre en scène un objet scientifique, tel qu'une nébuleuse en astrophysique. Il s'agit pourtant d'images qui cachent le fait qu'elles sont composées de milliers de visualisations préalables obtenues à travers plusieurs longueurs d'onde tout au long de plusieurs années de récolte de données - et ont été ensuite filtrées, recomposées, compressées, etc.

Regardons l'image d'un amas de galaxie, qui montre le fait qu' elle est composée de plusieurs données obtenues selon différentes longueurs d'onde (Figure 1). Elle est le résultat d'une composition/association/superposition/traduction de plusieurs images donnant l'illusion que ces amas de gaz peuvent produire un objet totalisant et compact (à gauche).

Si nous avons décrit l'image artistique comme autographique (unique [16], originale, immuable car sacralisant l'acte corporel non répétable de la fabrication), nous pourrions affirmer que le fonctionnement général de l'image scientifique la rapproche plutôt des systèmes symboliques dits allographiques où, comme c'est le cas en musique, une œuvre en tant que totalité se constitue par plusieurs exécutions. Cette totalité n'est d'ailleurs que transcendante, jamais matérialisée en un objet. Dans le cas des images scientifiques ainsi qu'en musique, il n'y a jamais de coïncidence entre l'objet (visuel ou sonore) et l'objet théorique transcendant constituant son
14. Sur les systèmes symboliques autographiques et allographiques, voir Nelson Goodman, Langages de l'art. Une approche de la théorie des symboles [1968], Paris, Hachette, 1990.

15. Pour une réflexion plus approfondie de cette question en relation avec la théorie de Bruno Latour sur la référence scientifique exposée dans plusieurs de ses ouvrages (dont Bruno Latour, L'Espoir de Pandore. Pour une version réaliste de l'activité scientifique, Paris, La Découverte, 2001), je me permets de renvoyer à Maria Giulia Dondero, "Sémiotique de l'image scientifique ", Signata Annales des sémiotiques/Annals of Semiotics 2010, $\mathrm{n}^{\circ}$ 1, pp. 111-174.

16. Certes, depuis la théorisation de Walter Benjamin sur l'opposition entre unicité et multiplicité et de Nelson Goodman sur l'œuvre d'art autographique et son unicité, le monde de l'art contemporain a grandement opéré de telle sorte à faire bouger ces distinctions et ces couplages catégoriels. À ce propos, voir Emilie Notteghem, "Frontières et franchissements. Les objets de culte catholique en artification ", in Nathalie Heinich et Roberta Shapiro, De l'artification. Enquêtes sur le passage à l'art, Paris, Éditions de l'EHESS, "Cas de figure ", 2012, pp. 47-62, où l'auteure montre qu'une des stratégies d'artification des objets religieux tels que les bénitiers passe par un processus de multiplication. "Ensemble, les bénitiers font œuvre d'art " (p. 56), car ils se détachent d'un usage personnel et dévotionnel pour devenir des objets de collection exposés à l'appréciation de l'expert. Sur le statut de la photographie entre statut autographique et allographique, voir Pierluigi Basso Fossali et Maria Giulia Dondero, Sémiotique de la photographie, Limoges, Pulim, 2011. 


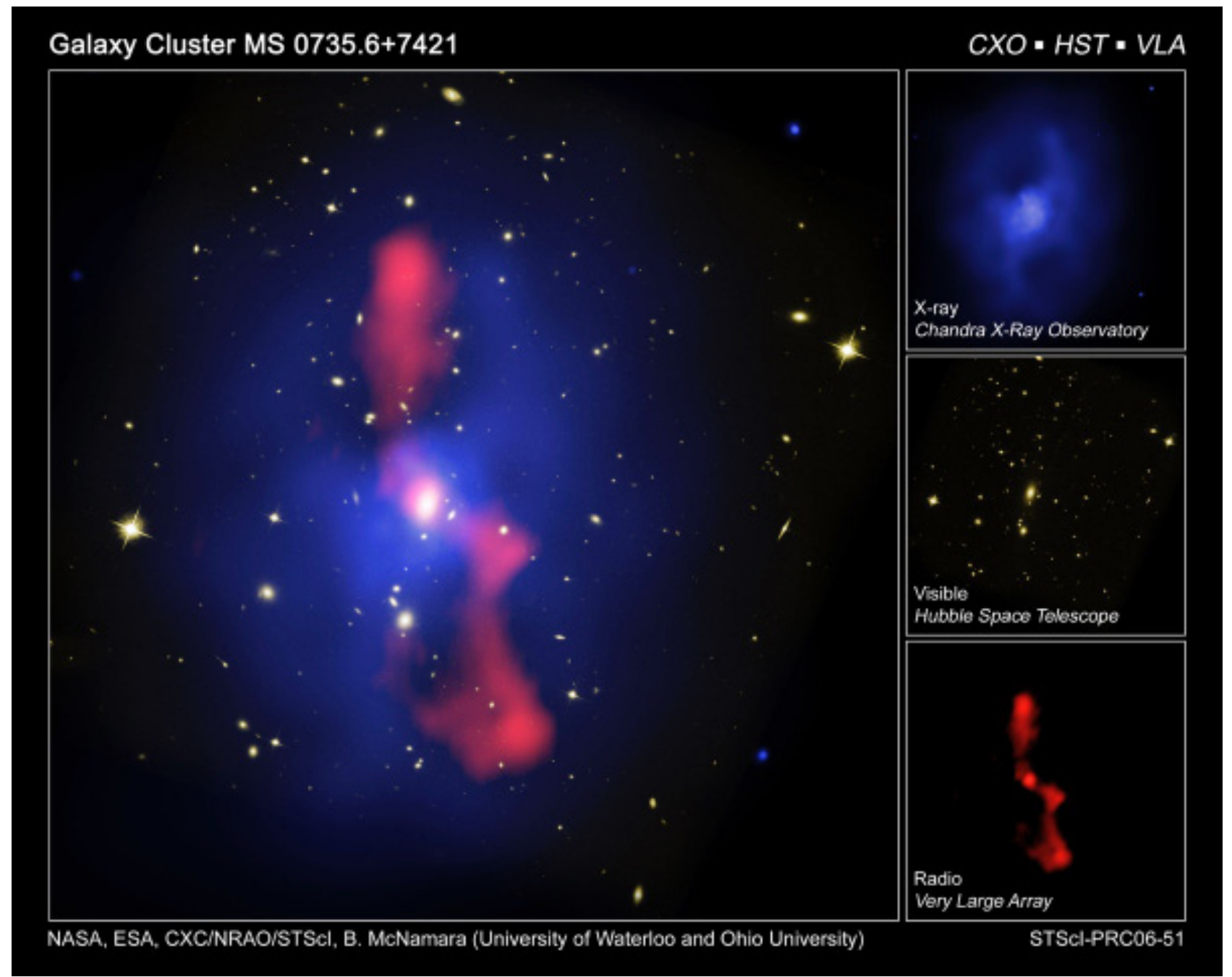

Figure 1. Galaxy Cluster MS 0735.6+7421. NASA, ESA, CXC/NRAO/STScl, B. McNamara (University of Waterloo and Ohio University). 
Voir en art, voir en sciences

identité (l'objet « nébuleuse », par exemple ou en musique l'objet « symphonie »). De plus, rien n'est immuable dans une chaîne d'images scientifiques : elles sont là en tant que produits de la manipulation et de l'expérimentation, prêtes à être répétées, vérifiées par d'autres chercheurs, et éventuellement falsifiées.

D'ailleurs, l'image isolée, obtenue par composition et superposition d'images, ne peut pas véritablement se prévaloir de l'adjectif «scientifique » car des images isolées figeant un objet théorique en une visualisation unique (et n'affichant pas le travail d'expérimentation qui les constitue) sont devenues des images de vulgarisation. L'image isolée de la chaîne de transformations qui l'a conçue, "sacralisée » ${ }^{[17]}$ en tant qu'identification de l'objet « nébuleuse ", se réduit à un produit immuable, non reproductible. Ce type d'images, où les paramètres de contrôle permettant les manipulations expérimentales ne sont plus ni affichés ni opérationnels, s'éloigne de l'allographie des images scientifiques expérimentales et se rapproche du régime de la vulgarisation ${ }^{[18]}$, proche des fonctionnements des objets artistiques, considérés comme « définitifs » et non manipulables.

Dans la pratique autographique de la peinture, par exemple, il existe plusieurs dispositifs de figement de l'acte pictural en objet fini, parmi lesquels on peut mentionner la signature, qui est l'étape qui finalise l'action de peindre [19]. La signature est d'ailleurs une prise de responsabilité, voire une attestation de sincérité/d'authenticité de la part de l'auteur ${ }^{[20]}$, et un engagement définitif. La signature est aussi le geste qui permet à l'œuvre d'être légitimée en vue de l'exposition future, à savoir de l'implémentation devant un public ${ }^{[21]}$.

La vulgarisation produit un travail d'autographisation de la chaîne d'images et l'offre à un large public. Dans l'image destinée à la vulgarisation, l'acte de fabrication, qui doit être connu et affiché dans la démarche expérimentale, reste caché ${ }^{[22]}$, rendant ainsi l'image non reproductible, comme dans le cas des images artistiques autographiques. Si la signature en art est non seulement un acte d'englobement (assomption de responsabilité) et donc, si j’ose dire, de cadrage, elle est aussi un acte d'exclusion (des esquisses précédentes, des ratures, des possibles futures contrefaçons, etc.). De manière similaire, la totalisation de plusieurs images scientifiques en une seule, offerte au regard d'un large public, est le résultat d'un processus de sélection et de muséification, voire de "séparation " des activités de recherche, de toutes sortes de tests et de vérifications. La vulgarisation est aussi un acte d'exclusion car elle englobe en une seule image la chaîne qui la compose, mais surtout elle exclut tout possible développement futur et collectif de cette image - les stratégies de l'allographisation telles que la notation visent en effet à transmettre des instructions d'exécution aux générations futures.

On peut également lire cette opération de suppression des paramètres d'expérimentation en tant qu'acte de recadrage et de re-méréologisation des composantes des différentes images en une image finale. Dans la figure 1, par
17. Nous parlons de sacralisation sur le modèle de l'image artistique en nous appuyant non seulement sur la sacralisation de l'original pictural mais aussi sur tous les cas de "séparation" et protection qu'engendre tout processus d'artification. À ce sujet, voir le cas des graffitis (de la rue à la toile) et de l'hip-hop (de la rue au théâtre) décrit dans Nathalie Heinich et Roberta Shapiro, "Postface. Quand y a-t-il artification ? ", in Nathalie Heinich et Roberta Schapiro, De l'artification. Enquêtes sur le passage à l'art, Paris, Éditions de l'EHESS, 2012, pp. 267-299.

18. Sur le rapport entre vulgarisation scientifique et images artistiques autographiques, voir Maria Giulia Dondero et Jacques Fontanille, Des images à problèmes. Le sens du visuel à l'épreuve de l'image scientifique, Limoges, Pulim, 2012.

19. Sur la signature dans la peinture, la sculpture et d'autres arts, voir Nathalie Heinich, " $\mathrm{La}$ signature comme indicateur d'artification ", Sociétés et représentations, 2008, $\mathrm{n}^{\circ}$ 1, pp. 97-106.

20. Sur la notion d'authenticité liée au point de vue de l'artiste (en rapport avec le point de vue de l'objet), voir Thierry Lenain, "L'authenticité ", in Nathalie Heinich, JeanMarie Schaeffer et Carole Talon-Hugon (dir), Par-delà le beau et le laid. Enquêtes sur les valeurs de l'art, Rennes, Presses universitaires de Rennes, «Aestetica », 2014, pp. 9-21.

21. Le cas de la signature comme acte d' " artification " contemporain permettant le passage à l'art de différentes activités culturelles et esthétiques par un processus d'individualisation est exploré tout au long de l'ouvrage de Nathalie Heinich et Roberta Schapiro, De l'artification. Enquêtes sur le passage à l'art, Paris, Éditions de l'EHESS, 2012.

22. Il faut pourtant préciser que les artistes contemporains affichent souvent les pratiques de production des objets artistiques, en les accompagnant ainsi de documents, photos de la production, écrits philosophiques, etc. D'une certaine manière, ces documents d'accompagnement finissent par s'identifier eux-mêmes avec l'œuvre, cette dernière ce " dispersant " dans un réseau de médias hétérogènes. Au sujet de l'artiste qui travaille à l'instar d'un scientifique qui collecte et expose les documents concernant toutes les étapes de la production (collective) de l'œuvre, voir Anne Beyaert-Geslin, "L'art comme texte et comme pratique de laboratoire ", in Anne Beyaert-Geslin et Maria Giulia Dondero (dir), Arts et Sciences. Approches sémiotiques et philosophiques des images, Liège, Presses universitaires de Liège, 2013, pp. 14-37. L'art parait alors dans ce cas aller vers un processus d'allographisation. 
exemple, cette re-méréologisation ne concerne que de simples opérations de superposition, mais dans d'autres disciplines, comme on le verra, les actions de totalisation peuvent être de différentes sortes.

Revenons aux chaînes d'images scientifiques expérimentales. Les images dans le discours scientifique de recherche nous offrent à chaque fois des " réponses partielles » qui se caractérisent comme des approximations et des tâtonnements successifs dont il faut tester la commensurabilité pour qu'on puisse les présenter, dans le cadre de la vulgarisation, comme identifiant une totalité d'objet théorique. D'ailleurs, c'est précisément la problématique de l'objet scientifique en tant que totalité à construire qui justifie dans notre travail le recours à l'outil de la rhétorique visuelle et de la méréologie. Cet outil vise à répondre à la question suivante : comment ces réponses partielles peuventelles construire une réponse globale afin de constituer et de stabiliser, au moins provisoirement, ce qu'on appelle un objet scientifique?

On pourrait affirmer que le parcours allant de l'objet en tant qu'interrogation jusqu'à l'objet scientifique fixé en tant que totalité acceptée - au moins par une partie de la communauté scientifique - se déploie à partir d'un état de questionnement en se dirigeant vers un état de partage communautaire et institutionnalisé ${ }^{[23]}$. Ce qui nous intéresse, c'est d'investiguer ce qui se passe entre ces deux moments, entre le questionnement et l'institutionnalisation d'un objet, à savoir le moment de la constitution iconique. J'entends par iconisation, en suivant la théorie de Jean-François Bordron ${ }^{[24]}$, les moments multiples des tests et des approximations visant à négocier et à stabiliser les formes d'un objet, en l'occurrence scientifique. Dans notre cas, l'iconisation peut se définir comme une instabilité méréologique de l'objet qui est en train de transformer un objet de recherches en un objet stabilisé et institutionnalisé. On n’entendra donc pas les images-tests en tant que signes iconiques au sens de ressemblant à

23. J'ai déjà traité la question de l'indicialité comme questionnement, de l'iconicité comme prise de formes et de la symbolicité comme médiatisation de l'objet de recherche au travers des conventions institutionnelles dans Maria Giulia Dondero, «The Semiotics of scientific image: from production to manipulation ", The American Journal of Semiotics, vol. 25, n 3-4, 2009, pp. 1-19.

24. Jean-François Bordron, « Rhétorique et économie des images ", Protée, 2010, vol. $38, \mathrm{n}^{\circ} 1$, pp. 27-39; L'Iconicité et ses images. Études sémiotiques, Puf, "Formes sémiotiques ", 2011 ; Image et vérité. Les dimensions iconiques de la connaissance, Liège, Presses universitaires de Liège, « Sigilla », 2013. quelque chose qui fonctionnerait comme un modèle préalable, mais en tant que compositions toujours partielles rendant compte de différentes organisations des données et des mesures. Dans le processus d'iconisation, les images qui testent l'objet de recherches afin de le stabiliser en un objet connu sont précisément à entendre comme des terrains d'expérimentation.

\section{LE CAS DE LA BIOLOGIE MOLÉCULAIRE}

Observons un premier exemple d'images concernant la question de la méréologie et des parties-tests de l'objet de recherches. Comme nous l'avons dit plus haut, les opérations rhétoriques et méréologiques dans le visuel se fondent sur le cadre et les jeux de cadrage. Nous analyserons ces opérations dans des séries d'images issues d'un article de biologie moléculaire, publié dans une des plus réputées revues d'immunologie, Immunity, en 2006. L'article examiné, qui a pour titre « Dynamics 
of thymus-colonizing cells during human development ${ }^{[25] ~ ", ~ p r e ́ s e n t e ~ u n e ~ g r a n d e ~}$ variété d'images consacrées aux différentes populations de cellules immunitaires censées coloniser le thymus avant et après la naissance du foetus ${ }^{26]}$.

Il est clair qu'aucune des images présentes dans l'article ne correspond à un objet singulier, à aucune totalité, que ce soit une cellule, une protéine, l'organe du thymus, ou autre chose. L'objet de recherches correspond en effet à une chaîne d'expériences, souvent trop longue pour être suivie en entier lors d'une publication. L'hypothèse que nous faisons ici pour limiter la chaîne pertinente à étudier est qu'une forme de totalité de l'objet scientifique est garantie par l'ensemble des images qui constituent l'article. On prend ainsi l'article comme totalité englobante et non pas toutes les expériences sur ce phénomène dans leur ensemble. Les images sélectionnées pour la publication sont par conséquent déjà le résultat d'un cadre dans le cadre, ou plutôt d'un arrêt sur image à l'intérieur de la dynamique d'un flux, voire d'un processus expérimental très long.

Au début de l'article étudié, l'objet de recherche est encore virtuel et il se constitue discursivement au fur et à mesure des manipulations expérimentales des scientifiques. Àl'intérieur de l'article, l'objet de recherches subit des découpages, des manipulations, devient ainsi élastique. Les visualisations nous montrent les transformations du processus lui-même ou, plus précisément, la manière de le découper, spatialement et temporellement, et ainsi de l'analyser. Comme nous l'avons évoqué plus haut, l'image possède comme première caractéristique celle d'encadrer, de sélectionner, de découper, voire d'isoler des parties et de focaliser l'attention de l'observateur. Ce fonctionnement est en harmonie avec une des opérations les plus fondamentales en biologie qui est celle d'arriver, via des modèles, à isoler des problèmes, à repérer des bords à l'intérieur du flux de la vie en perpétuelle transformation.

Si l'ensemble d'images publiées dans l'article constitue déjà un " cadre dans le cadre » dans sa globalité par rapport au flux expérientiel du phénomène à étudier, la série d'images de la figure 2, qu'on trouve à la page 226 de l'article examiné, met en revanche en scène un premier type de cadre dans le cadre interne au cadrage global. On est face ici à différents types de cadrage visant la recherche de la cellule censée coloniser le thymus (Figure 2).

Dans les deux premières images disposées en haut (groupe A), nous visualisons quatre petits rectangles situés aux bords de la forme colorée en vert; ils nous permettent de localiser les zones qu'il faudra ensuite focaliser, sur lesquelles nous devrons centrer l'attention et ainsi poursuivre l'exploration, voire l'approfondir.

Les quatre images du groupe $\mathrm{B}$ que l'on trouve en dessous des deux premières $(\mathrm{C} 1$, C2, C3, C4) sont en effet engendrées par les deux d'en haut par focalisation et zoom. Ces quatre images nous permettent d'obtenir non seulement une meilleure vision/ focalisation en termes de définition de la qualité de vision (la vision microscopique
25. Haddad et alii, « Dynamics of thymuscolonizing cells during human development ", Immunity, 24, pp. 217-230, février 2006.

26. Le point de la recherche en très bref : toutes les cellules du système immunitaire se développent dans la moelle osseuse sauf les lymphocytes T, qui se développent dans le thymus. Le thymus est colonisé par des cellules produites dans la moelle osseuse par voie sanguine : il s'agit de comprendre quelle est la cellule qui rentre dans le thymus. Une cellule souche, indifférenciée, rejoint-elle le thymus par un parcours aléatoire, ou bien les cellules colonisant le thymus sont-elles prédéterminées à y aller ? On sait que la différenciation cellulaire commence dès le stade de la moelle osseuse, mais comment repérer les cellules qui sont censée être préprogrammées, voire dédiées sélectivement à la colonisation de l'organe du thymus ? À ce sujet, nous avons réalisé un séjour de recherche à l'Institut universitaire d'Hématologie (Paris 7) en 2014 sous la direction de Bruno Canque. Nous tenons à remercier Bruno Canque et Kutaiba Alhaj Hussen pour les explications et les échanges généreux. 
A

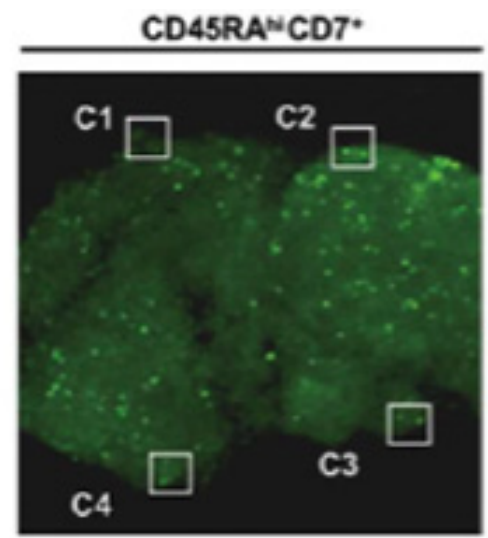

B
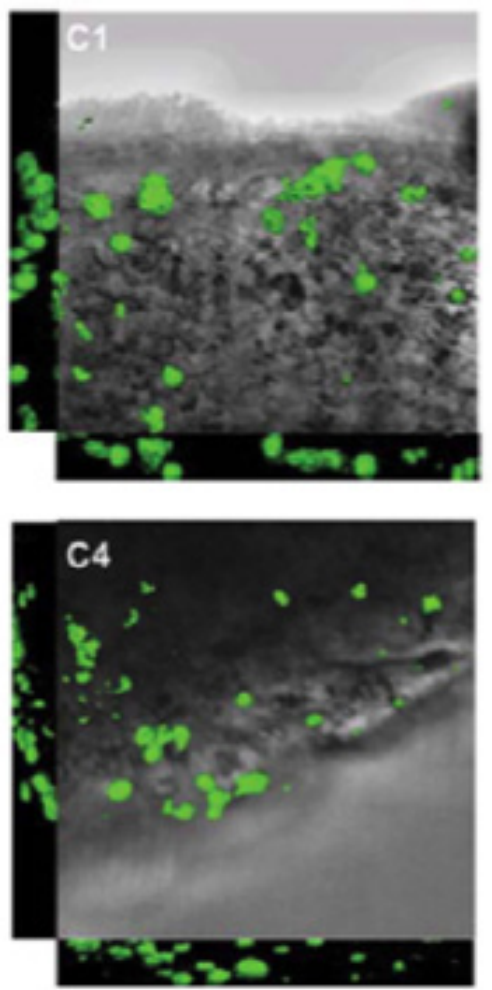

CD45RA ${ }^{\text {int }} \mathrm{CD}^{\circ}$.
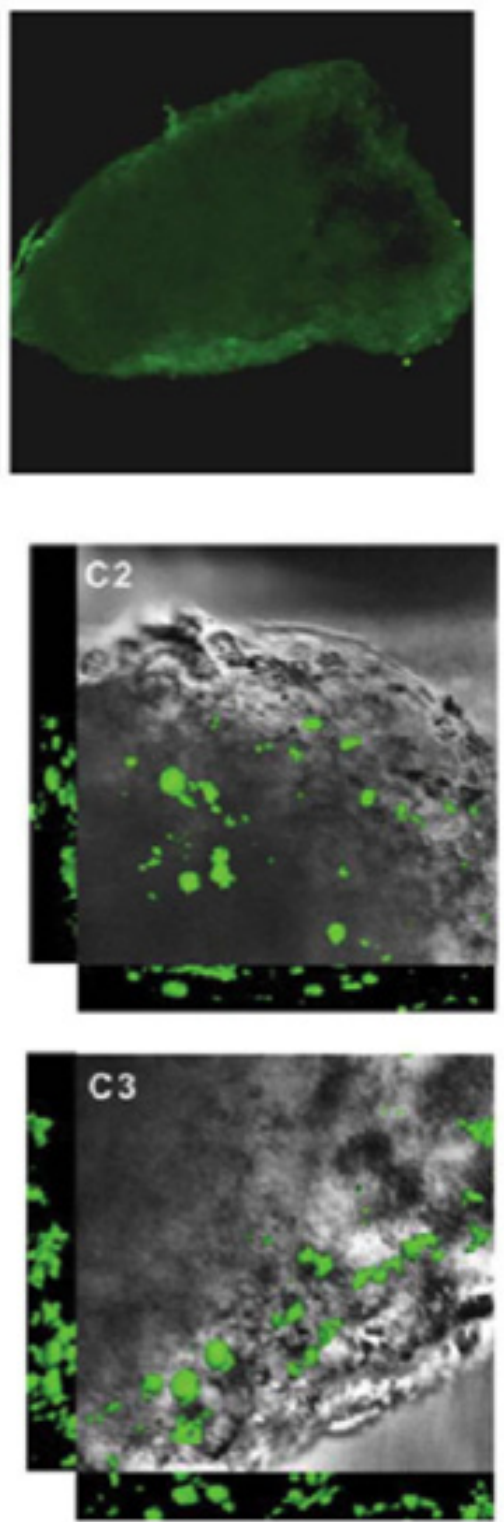

Figure 2. «Ex Vivo Thymus Colonization Assay» (Haddad et alii, 2006 : 226). 
est plus avancée), mais elles permettent véritablement une ouverture sur une nouvelle scène, sur un processus dans le processus.

Ce fonctionnement pourrait être rapproché de celui produit par le dispositif de la fenêtre (ces cadres sont d'ailleurs appelés fenêtres par les biologistes). Si dans les images artistiques, et notamment des paysages, la représentation de la fenêtre renvoie à une ouverture vers l'altérité, une ouverture de l'intérieur vers l'extérieur, dans l'image en biologie la fenêtre fonctionne comme une ouverture vers l'intériorité de l'objet et, plus généralement, comme l'indicateur d'un processus de projection en avant de la recherche, d'un acte de devancement : d'autres images suivront. Ce processus engendré par le dispositif de la fenêtre dans le genre du paysage concerne le passage du connu (l'intérieur d'où nous regardons) à l'inconnu (l'extérieur qui s'ouvre devant nous) : c'est le même passage qui concerne l'engendrement des images du groupe B au départ du groupe $\mathrm{A}$.

Ces focalisations fonctionnent aussi comme des dispositifs de multiplication de nouvelles scènes qui peuvent à leur tour offrir l'accès à des scènes ultérieures, par l'ouverture d'autres fenêtres. Ces images creusent à l'intérieur de l'objet à analyser et en même temps ouvrent sur de nouveaux terrains, de nouvelles visions.

En parallèle, l'opération de focalisation d'une partie du flux engendre en même temps la soustraction d'une zone par rapport au fond, le fond étant le lieu de l'image où il n'y a pas d'acteurs de la transformation. Dans C1, C2, C3 et C4 (groupe B), nous avons, d'un côté, gagné en profondeur de la vision par rapport aux images du groupe A, en intensité pourrait-on dire, mais nous avons, de l'autre, perdu l'étendue de la vision que nous avions obtenue via les deux premières images du groupe A. Les nouvelles images ont provisoirement perdu le fond qui était constitué, dans les images d'en haut, du tissu coloré en vert.

Nous découvrons ainsi avec $\mathrm{C} 1, \mathrm{C} 2, \mathrm{C} 3$ et $\mathrm{C} 4$ un autre type d'encadrement, qui fonctionne de manière différente de l'encadrement "à fenêtre " : il s'agit d'un encadrement par superposition. C1, C2, C3 et C4 se manifestent à travers une action de superposition sur un nouveau fond (taches vertes sur fond noir) qui est caché mais qui, en même temps, émerge. C1, C2, C3 et C4 mettent en valeur (par des taches vertes sur fond gris) une population de cellules qui, après excitation, permettra à certaines protéines de bien se fixer sur l'organe du thymus.

Mais comment expliquer le fonctionnement des fonds noirs sur lesquels émergent des fonds gris clair, sur lesquels émergent à leur tour les concrétions en vert obtenues par fluorescence? C'est ce fond noir en partie inaccessible qui permet l'émergence du fond gris qui fait face à l'observateur et qui s'affiche comme quelque chose doté d'un degré de stabilisation iconique plus marqué - car en saillie - que ce qui se passe dans le fond noir. 


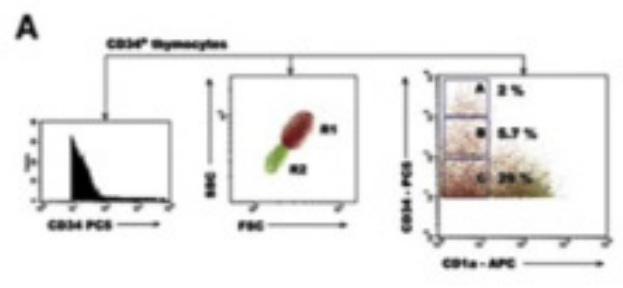

B

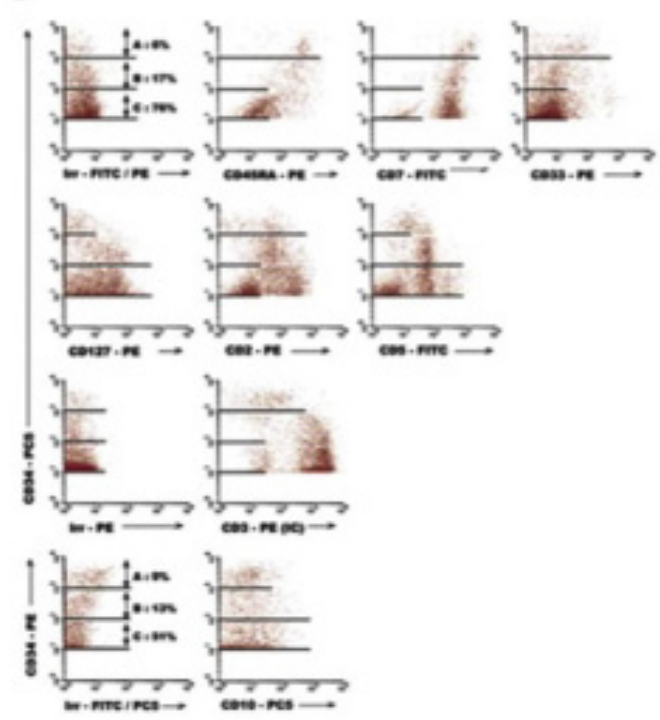

C

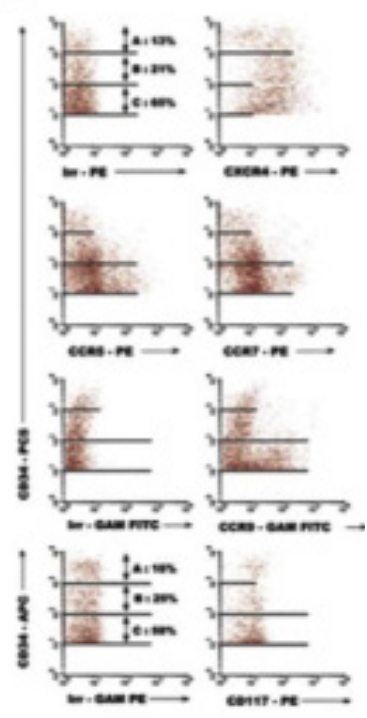

Figure 3. «Phenotypic and Molecular Characterization of Immature CD34+CD1a2/+ Fetal Thymocytes » (détail, Haddad et alii $2006: 223$ ). 
Il faut aussi remarquer que chaque groupe d'images (A et B) expose un double cadre : dans les deux premières images (groupe $\mathrm{A}$ ), nous sommes face à une totalité produite par le tissu vert, qui est en attente de focalisation des quatre parties sélectionnées par les carrés - ce qui amène à une opération de soustraction de la totalité. Les images $\mathrm{C} 1, \mathrm{C} 2, \mathrm{C} 3$ et $\mathrm{C} 4$ sont précisément les produits de l'extraction d'une totalité provisoire. Elles ont également besoin d'un cadre comme base fonctionnant, d'un côté, comme mémoire de la totalité de laquelle elles ont été extraites et, de l'autre, comme présentoir affichant ce que les nouvelles observations par focalisation nous permettent de voir.

Les images d'en haut (groupe A) hébergent des cadres qu'on pourrait appeler protensifs, c'est-à-dire des cadres allant vers l'avenir de l'expérimentation (le groupe B), tandis que les images d'en bas (groupe B) recherchent en revanche un ancrage dans le groupe A : elles se superposent sur des fonds qui leur servent de mémoire. Ces derniers types de cadrages peuvent être appelés retensifs.

Nous pourrions affirmer en effet que les images du groupe A sont en attente, elles préfigurent d'autres images potentielles, des images à venir; elles prévoient en somme un mouvement en avant, portées qu'elles sont par une sorte de séquentialité. En revanche, les images du groupe B sont une sorte de déploiement/ approfondissement des premières, dont elles gardent soigneusement les traces. C1, C2, C3 et C4 gardent en mémoire la trace d'une sorte de micro-totalité qui les a produites. Elles engendrent un mouvement en avant et en arrière. Ce qui est significatif ici est que les actes d'encadrement peuvent signifier plusieurs sortes d'opérations, plus ou moins protensives ou rétensives.

Dès ces premiers exemples, la pertinence de la rhétorique méréologique formulée par Jean-François Bordron dans sa théorie de l'iconicité devient évidente. Cette rhétorique est en fait entendue en tant que gestuelle constituant des morphologies à partir d'un flux expérientiel : cette gestuelle - qui est en effet une action de manipulation - opère sur des totalités et sur des parties, voire sur une hiérarchie de combinaisons et de sélections de cadrages constituant les expériences. Il s'agit de supposer que l'objet des recherches est doué d'une dynamique susceptible de compositions multiples.

Le fonctionnement d'ouverture à fenêtre est repérable dans cette autre chaîne d'images (Figure 3) qui, à la différence de l'autre, n’ouvre pas de nouvelles scènes mais effectue des sélections et des tris.

En effet, dans la série $\mathrm{A}$, la toute première image à gauche, qui fait partie d'une série de trois images, met en scène une population de cellules sélectionnées (cellules étant susceptibles de coloniser le thymus), tandis que la deuxième image montre les différentes tailles dont sont faites les cellules de cette population. La troisième image trie encore, car elle ne rend compte que du développement 


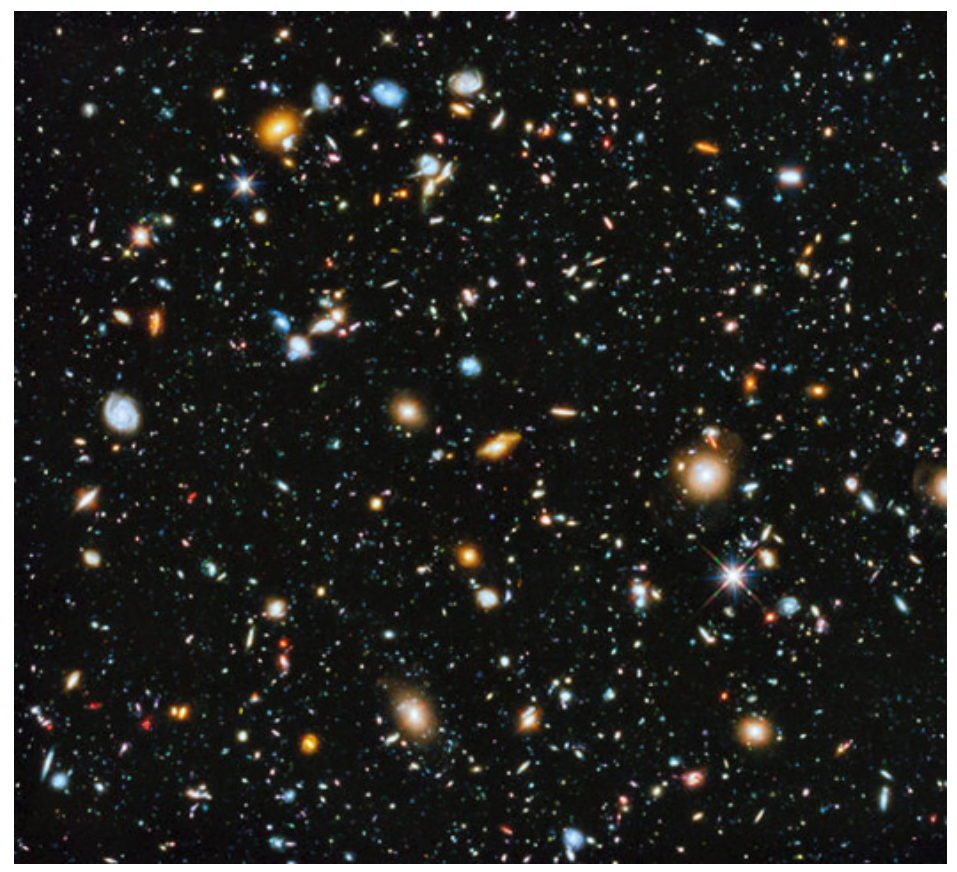

Figure 4. Image représentant 10000 galaxies, produite par combinaison de 841 photos ayant enregistré la totalité du spectre lumineux observable par le champ ultra-profond du télescope. | NASA, 2014.

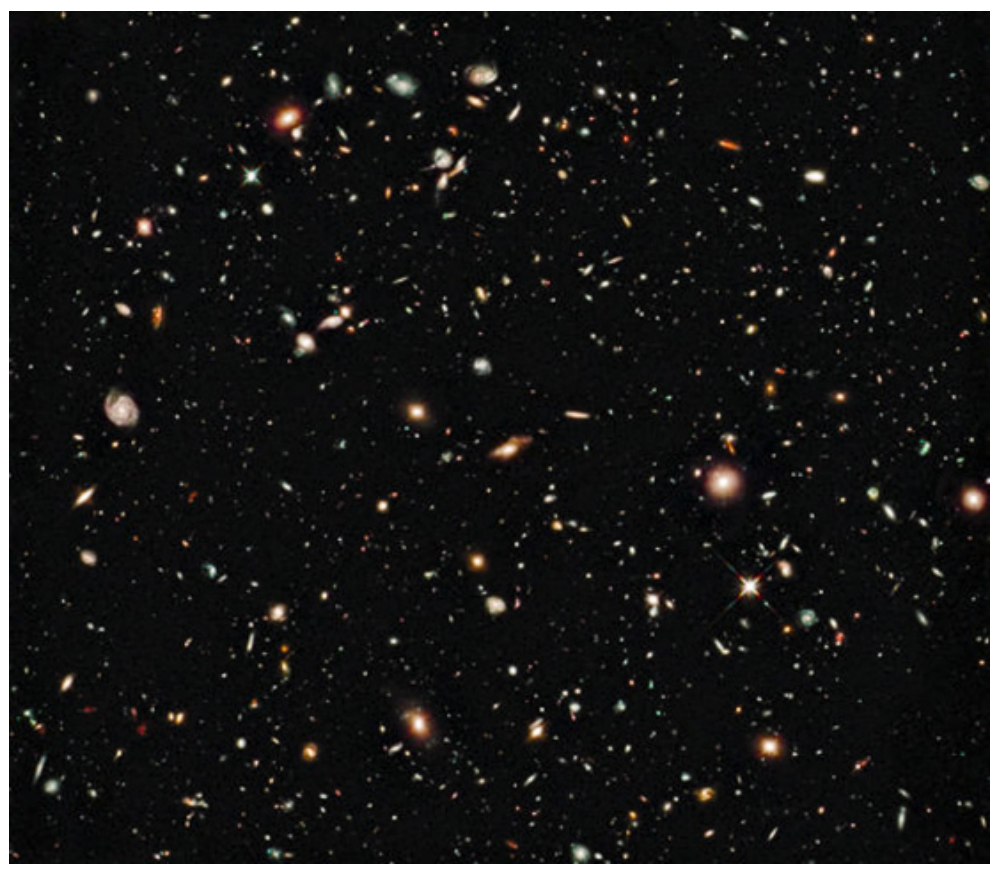

Figure 5. NASA, 2009. 
temporel des cellules d'une seule taille, représentées en rouge. Cette méthode d'enchaînement d'images ne concerne pas des opérations de focalisation ou de superposition, mais bien de division et de tri.

Cette méthode de tri ne se rapproche de celle des portes et des fenêtres que partiellement ; en effet, ici il ne s'agit pas de soustraire de la puissance de visibilité dans un champ de vision, mais bien de trier et de diviser, opération plus rare dans le cadre des images artistiques - sauf quand il s'agit de tableaux-expérimentations sur le fonctionnement de la lumière, comme dans les cas des tableaux de Sonia et Robert Delaunay, par exemple, ou encore d'études sur la décomposition du mouvement chez Duchamp ou chez Boccioni et les Futuristes italiens.

Dans Nu descendant un escalier de Duchamp, par exemple, l'opération de tri concerne la décomposition du mouvement d'un sujet humain en ses composantes. Les composantes du corps humain ne peuvent pas correspondre aux composants du mouvement de ce même corps. Ce que l'œuvre met en scène est justement une imperfection de correspondance : la composition des parties constituant un corps est en train d'éclater, et en même temps, la forme du mouvement ne s'est pas encore émancipé de la forme totalisante du corps, qu'on peut encore partiellement repérer en creux.

Dans le cas de l'image scientifique, l'action de trier est plus nette et tranchée car la matière sur laquelle on opère a déjà subi des segmentations et des discontinuités préalables ; la chaîne qui la porte est, pourrait-on dire, un continuum de discontinuités. D'ailleurs c'est aux images artistiques qu'il revient de mettre en scène des imperfections ou des incertitudes qui ne demandent pas à être résolues. En effet, la matière picturale sur et à travers laquelle on opère les segmentations est dense, saturée syntaxiquement et sémantiquement comme toute œuvre autographique. Ce n'est d'ailleurs pas un hasard que dans plusieurs disciplines et notamment en astrophysique, toutes les images qui ne peuvent pas être falsifiées sont appelées "vues d'artistes ", bien que produites par des scientifiques. Les sciences recourent à l'étiquette artistique lorsque la visualisation ne s'appuie ni sur des socles mathématiques ni sur des données vérifiables ; d'une certaine manière, l'art fonctionne ici comme promesse de développement scientifique futur ${ }^{[27]}$.

\section{LE CAS DE L'ASTROPHYSIQUE ET DE L'ARCHÉOLOGIE}

Venons-en à d'autres images, notamment dans le cadre de l'astrophysique. L'astrophysique fonctionne plutôt par addition de points de vue, obtenus par enregistrement de la lumière selon différentes longueurs d'onde, comme nous l'avons vu précédemment (Figure 1) et comme nous le voyons également dans cette image représentant 10000 galaxies (Figure 4). Cette image a été publiée dans
27. Voir à ce sujet : Maria Giulia Dondero, "L'argumentation de l'image scientifique. Une affaire de méréologie ", in MarieChristine Pollet (dir.), Argumenter dans les écrits scientifiques, Diptyque, sous presse, pp. 51-68. 


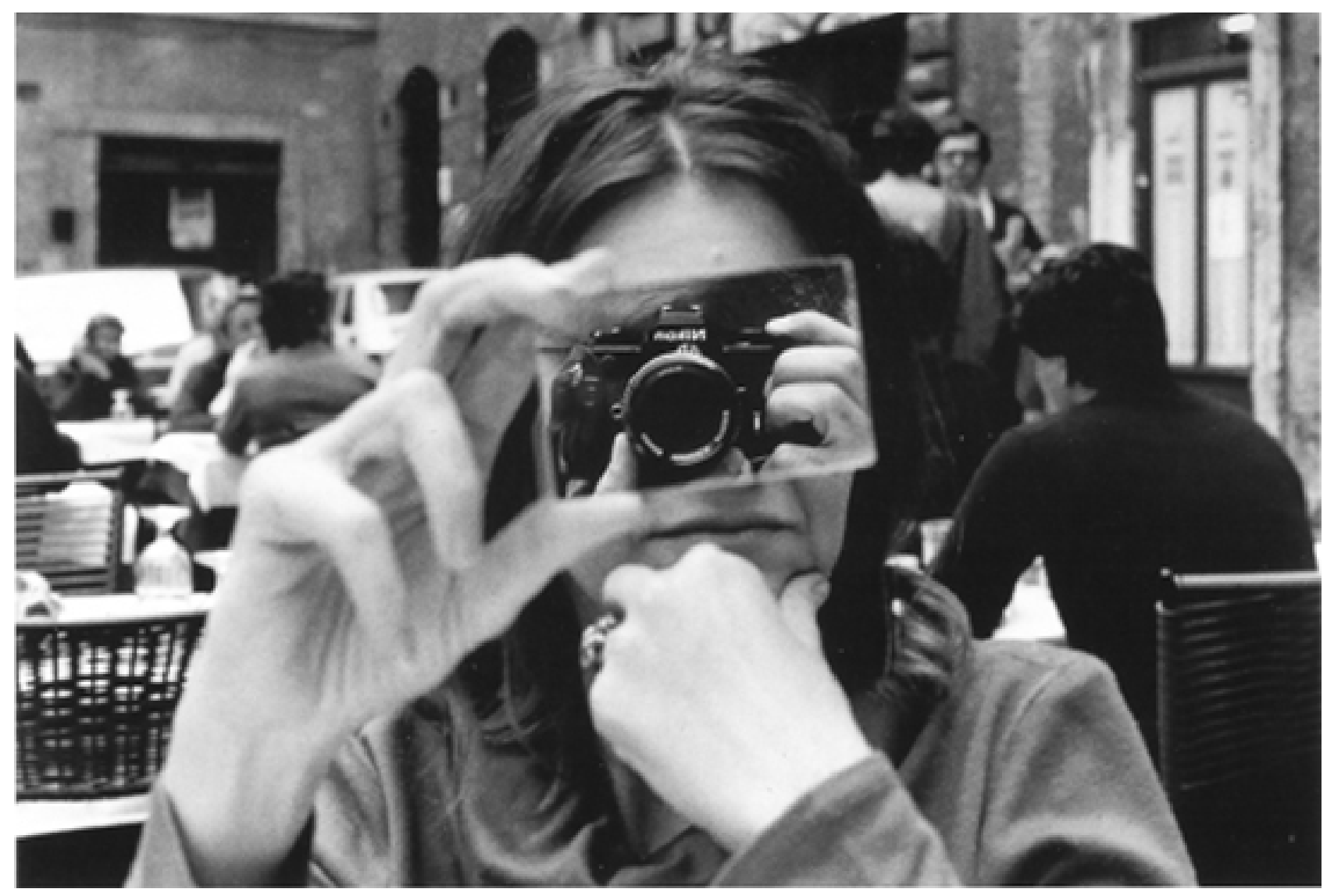

Figure 6. Denis Roche, 28 mai 1980 (Rome, « Pierluigi »), by kind permission of Galerie Réverbère, Lyon. 
la rubrique «Sciences » du Monde en mars 2014 et « ajoute » un point de vue sur ce qui avait été enregistré en 2009 (Figure 5). La photo de 2014, par rapport à celle de 2009, ajoute la vision en ultraviolet qu'il n'était pas possible d'obtenir en 2009.

Chaque longueur d'onde peut être entendue comme une sorte de cadrage, une manière différente de focaliser et tester la puissance de vision de différentes technologies impliquées dans l'exploration des astres.

Nous avons parlé d'addition de points de vue pour illustrer les opérations caractérisant le miroir : le miroir permet en effet d'englober, à travers son cadre dans le cadre, des objets et des parties de l'espace phénoménologique préalablement laissés hors du champ de vision. Le miroir intègre différents points de vue, notamment des points de vue complémentaires. L'histoire de la peinture et l'histoire de la photographie sont parsemées d'exemples majeurs de cette inclusion de points de vue hétérogènes encastrées au sein du point de vue dominé par la perspective frontale. En plus des études de Stoichita sur des tableaux tels que Las Meninas de Velázquez ou Les époux Arnolfini de van Eyck, nous pouvons rappeler la production d'un photographe tel que Denis Roche qui a su superposer le point de vue du sujet photographié au point de vue du sujet photographiant. Je me limiterai à rappeler le cas de la photographie 28 mai 1980 (Rome, «Pierluigi »), qui met en scène une véritable « bataille de dispositifs " ${ }^{[28]}$ (Figure 6).

La femme, objet de la prise de vue, devient elle-même co-auteure de la photographie en substituant à la prise de son regard un miroir qui lui permet de déclencher une autre perspective, voire une autre vision (la vision de la production). À travers ce renversement du point de vue, une nouvelle perspective entre en concurrence avec celle de la prise de vue principale. Il s'agit d'un conflit de perspectives : le miroir est utilisé comme résistance de la part de la femme qui refuse partiellement d'apparaître dans la photo en tant que modèle, en tant qu'objet de la prise de vue, et devient ainsi elle aussi regardeuse - et non seulement regardée -, ainsi que productrice d'une vision sur la vision qui l'engendre.

À la différence de la peinture et de la photographie artistiques pourtant, l'insertion d'un nouveau point de vue dans une image d'astrophysique ne produit pas une multiplicité hétérogène mais engendre une homogénéisation totale des multiples traces lumineuses.

Passons à un autre type de fonctionnement de l'image scientifique en nous intéressant cette fois aux stratégies de fabrication des images en archéologie et notamment à celles produites à travers les méthodes géophysiques des projections électromagnétiques.

Les images en archéologie sont aussi construites sur des superpositions de perspective et elles mettent notamment en rapport les images-mesures de la
28. Pour une analyse plus approfondie de cette photo et de la série à laquelle elle appartient, voir Maria Giulia Dondero et Pierluigi Basso Fossali, Sémiotique de la photographie, Limoges, Pulim, 2011. 


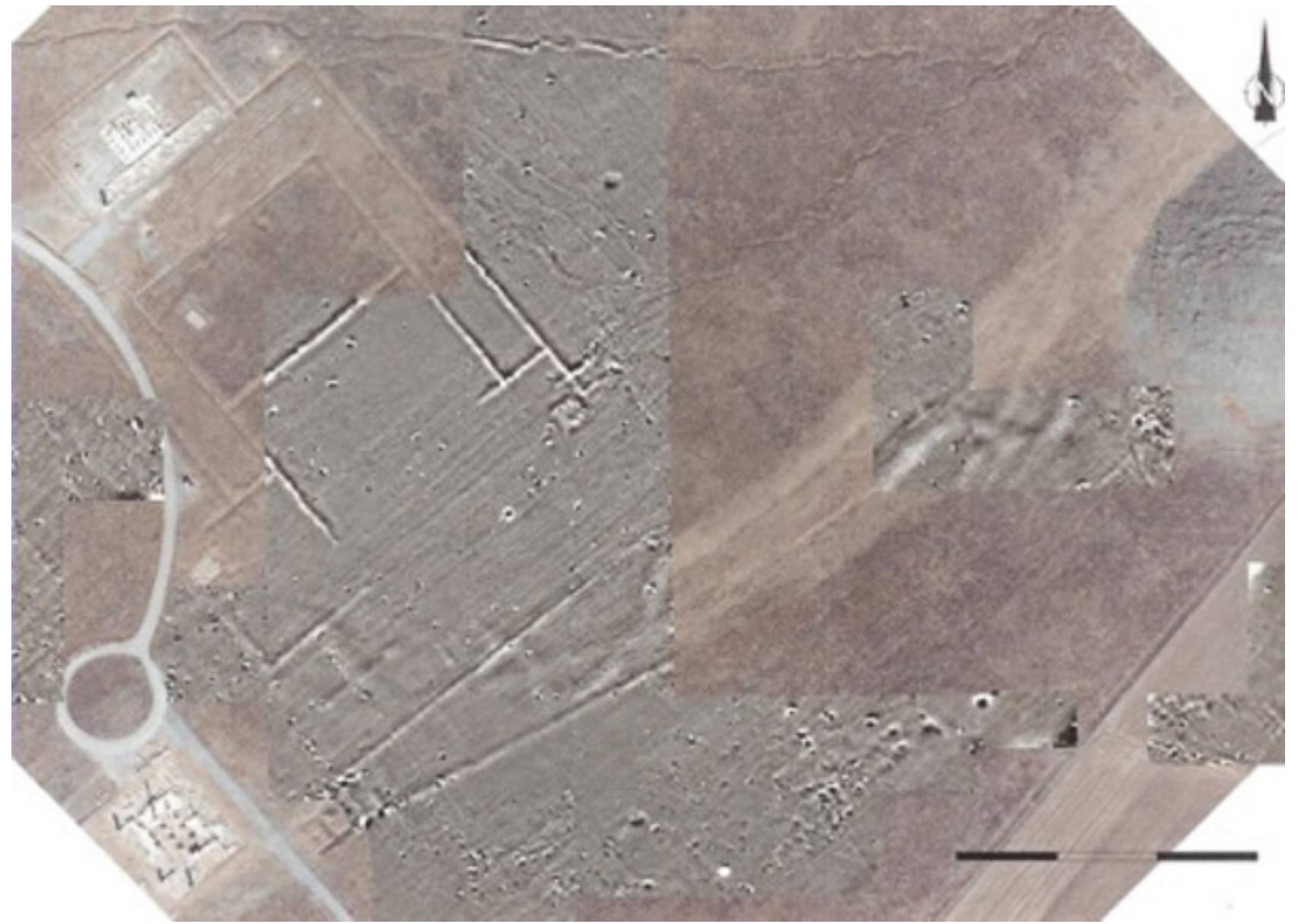

Figure 7. Prospections magnétiques autour des palais de rois perses, superposées aux photos aériennes par le centre archéologique de Persépolis (image publiée dans Dossiers d’archéologie, ${ }^{\circ} 308$ (2005 : 11)). 
prospection électromagnétique avec les images produites par la prospection aérienne (Figure 7), où l'hétérogénéité des méthodes de détection, contrairement à l'astrophysique, permet non pas une intégration mais plutôt ce qu'on pourrait appeler une intravision diagrammatique ${ }^{[29]}$.

Dans cette image on a affaire à deux syntaxes figuratives juxtaposées : l'intravision diagrammatique est un interstice entre deux systèmes de pertinence, entre deux régimes de regard. L'intravision diagrammatique est une forme de visibilité nouvelle, produite par la transposition réciproque de deux régimes du voir et du mesurer par rapport auxquels on cherche une commensurabilité. Il s'agit d'une vision interstitielle qui permet de percevoir une possible transponibilité de relations d'un système de représentation à l'autre.

Cette commensurabilité envisagée est supportée sur le plan de l'expression par des réseaux de relations qui se caractérisent de manière contrastive et qui permettent de construire sur le plan du contenu des valeurs différentielles. Mais il ne s'agit pas d'un simple isomorphisme entre les plans d'expression. L'intravision diagrammatique est au contraire dynamique parce qu'elle permet de reconstruire des patterns en évolution, saisir des syntaxes, à la fois sur le plan de l'énoncé (processus des événements et des objets observés) et sur le plan de l'énonciation (processus d'investigation).

\section{Intégration et intravision diagrammatique}

Comme nous venons de le voir, les images en astrophysique visent à additionner, superposer, intégrer - phagocyter presque - les traces lumineuses, voire à réduire l'hétérogénéité des captations dans une seule image, comme dans l'exemple de l'amas de galaxie (Figure 1), qui montre la manière dont l'image finale intègre les images provenant des différentes détections afin de stabiliser l'identité d'un objet. Dans l'image de l'amas de galaxie, tout se passe en fait comme si les visualisations qui la composent possédaient une consistance " transparente » envers les autres images, le but final étant une superposition qui homogénéise les traces des captations différentes. Nous obtenons ainsi une image de la transparence, voire une image construite sur la composition de perspectives et pertinences différentes (fonctionnement du miroir selon Stoichita). Il paraît en effet que l'image finale est destinée à compacter les visualisations partielles, voire ce qui dans l'univers est dispersé ; elle vise ainsi à construire des simulacres de cohésion de ce qui en réalité est diffus dans le temps et dans l'espace. L'image finale a assimilé toutes les mesures : c'est dire que le point d'arrivée est l'homogénéisation de traces hétérogènes.

Or, tout cela est loin d'être vrai pour l'image en archéologie, où une mesure et une méthode de détection ne sont jamais intégrées ni totalement intégrables à l'autre. En effet, les images dans cette discipline ne visent pas l'intégration des
29. Dans la définition de diagramme on s'appuie sur la théorie de Charles Sanders Peirce qui distingue ainsi le diagramme de l'image et de la métaphore : " On peut en gros diviser les hypoicônes suivant le mode de la priméité à laquelle elles participent. Celles qui font partie des simples qualités ou premières priméités sont des images ; celles qui représentent les relations, principalement dyadiques ou considérées comme telles, des parties d'une chose par des relations analogues dans leurs propres parties, sont des diagrammes ; celles qui représentent le caractère représentatif d'un representamen en représentant un parallélisme dans quelque chose d'autre sont des métaphores " (Peirce, Collected Papers of Charles Sanders Peirce, 8 vol., numérotés en chiffres arabes, Hartshore, Weiss, Burks dirs., Cambridge, Harvard University Press, 1931-35, 2.276-7). À ce sujet, voir aussi l'ouvrage fondamental de C. Chauviré, L'Eil mathématique. Essai sur la philosophie mathématique de Peirce, Paris, Kimé, 2008. 
traces : tout au contraire, elles mettent en scène l'hétérogénéité des méthodes de recherche, leur impossibilité de se recomposer en unité et de visualiser des unités d'objets (fonctionnement de la porte et des rideaux selon Stoichita). Cette impossibilité d'homogénéisation permet non seulement une double ou une multiple vision du même événement, mais surtout une investigation qui se fonde sur la dissimilation des traces. Chaque image en archéologie doit montrer la diversité des méthodes de mise en relation des surfaces avec le sous-sol, ou des différentes parties du sous-sol ; chaque méthode d'enquête met en scène un processus d'exfoliation de quelque chose de compact. L'image entend signifier les méthodes elles-mêmes de la recherche en archéologie et notamment que chaque système de captation et de représentation fonctionne par " prélèvement successif » de toutes les couches stratifiées dont le sous-sol est constitué : les stratégies de captation et de représentation visent à dissimiler les stratifications. En somme, la fonction des images en archéologie est tout à fait différente de celle qu'elles assument en astrophysique : à partir du compact, il faut parvenir à une exfoliation du sous-sol, en montrant toute la diversité dont il est constitué. Chaque couche doit en montrer une autre, ou plusieurs autres : c'est par la dissimilation que l'on peut parvenir à voir et opérer. C'est ce même processus que l'image est censée mettre en évidence. Nous avons rapproché ce fonctionnement des opérations mises en œuvre par les rideaux et par les portes que Stoichita décrit comme caractéristiques des peintures d'intérieur du réalisme du Siècle d'Or hollandais, et notamment des peintures de Vermeer et de Maes. Ces tableaux qui montrent des actes intimes tels que l'écriture/lecture d'une lettre d'amour mettent en scène un conflit entre une focalisation sur l'acte de l'écriture/lecture et les obstacles qu'il faut franchir pour que cette focalisation se déploie. Les portes et les rideaux, dispositifs du voir à travers, sont des exemples d'une composition de points de vue qui permet une vision double qui ressortit du dépassement d'un obstacle. Nous les avons décrites ailleurs ${ }^{[30]}$ comme des stratégies de l'accessibilité à la vision : il ne s'agit pas d'une impossibilité de voir (inaccessibilité), ni d'une exposition offerte au regard de l'observateur mais bien d'une intravision qui demande à être déployée. C'est ce qui se passe en effet dans les images d'archéologies générées par des méthodes de la géophysique (non-invasifs) : dépasser l'obstacle de la compacité du sol et du sous-sol, qu'on ne peut pas détruire, mais dont il faut faire émerger l'hétérogénéité et la stratification des traces.

\section{POUR CONCLURE}

30. Maria Giulia Dondero, « L'énonciation énoncée dans l'image ", in Marion ColasBlaise, Laurent Perrin et Gian Maria Tore, L'Énonciation aujourd'hui, Limoges, Lambert Lucas, 2016, pp. 238-255.
Les images en astrophysique et en archéologie présentent deux types différents de virtualité de l'objet de la perception : l'objet de l'astronomie est absent à nos sens, caché, parce qu'il est trop diffus et lointain, distal, alors que l'objet archéologique est caché parce que sa stratification est trop compacte. Il s'ensuit qu'en astrophysique les images actualisent l'objet par des assimilations qui compactent l'évanescent et qui visent justement la représentation de certains 
phénomènes en les transformant en une «totalité d'objet », alors qu'en archéologie les images fonctionnent par dissimilation de ce qu'elles actualisent, c'est-à-dire par dissimilation au sein d'une matière stratifiée et compacte. En astrophysique, on opère par superposition et intégration de modules de lumière; en archéologie, en revanche, par différenciation de couches. Si le but de l'astrophysique est de visualiser des phénomènes en les représentant en tant qu'objets unitaires, l'archéologie s'appuyant sur les méthodes de la géophysique vise par contre la représentation d'une tension de forces pouvant révéler les rapports réciproques entre couches temporelles appartenant à différentes strates du sol (intravision diagrammatique).

Enfin, en suivant notre schématisation, on peut conclure que l'image en astrophysique fonctionne surtout comme un miroir (addition/intégration des points de vue), tandis que l'image en archéologie fonctionne surtout selon le modèle des portes et des rideaux (intravision).

En ce qui concerne les images en biologie, nous avons en revanche remarqué une certaine variété de dispositifs : si les opérations les plus fréquentes sont des ouvertures à fenêtre, on peut également repérer des fonctionnements suivant le schéma des niches, à savoir la constitution d'un équilibre entre des forces de barrage de l'horizon et d'émergence de formes vers l'observateur (rétension et protension).

En fin de parcours, nous pouvons affirmer que la transposition des dispositifs métasémiotiques via les opérations de cadrage a permis de mettre en valeur les opérations rhétoriques de manipulation de l'objet scientifique ainsi que les différentes stratégies de composition de la totalité.

Certes, la distance entre les images artistiques qui ont dirigé notre attention sur ces opérations rhétoriques et les images scientifiques examinées, est importante, surtout si l'on compare ces images d'un point de vue strictement figuratif. Les différences sont aussi significatives si nous envisageons ces images du point de vue de leur statut social, à savoir du point de vue de l'économie des valeurs qui les rendent interprétables. L'art relève de la contemplation d'objets uniques et "protégés ", tandis que la science relève d'actions de manipulation et transformation. Pourtant, on a pu constater une correspondance évidente entre les opérations de composition/décomposition de la matière du visible en art et dans les exemples scientifiques choisis, ce qui nous permet de repérer des fonctionnements communs dans le processus d'argumentation. 